\title{
Alternating exposure to two compound flavors creates inhibitory associations between their unique features
}

\author{
D. M. DWYER \\ Cardiff University, Cardiff, Wales \\ and \\ N. J. MACKINTOSH \\ University of Cambridge, Cambridge, England
}

\begin{abstract}
Rats were exposed to two compound solutions, saline-lemon and sucrose-lemon. In Group ALT, trials with one solution alternated with trials with the other. Group BLK received all trials with one solution before any trials with the other. Previous retardation tests had implied that only alternating exposure would establish sucrose as an inhibitor of saline. To provide a complementary summation test for this inhibition, in Experiment 1, all the animals received pairings of peppermint and saline and were tested for consumption of peppermint-sucrose under sodium depletion. Consumption was increased by sodium depletion only in Group BLK. In Experiment 2, a retardation test was used to show that presentation of saline-lemon before sucrose-lemon on each exposure day would establish sucrose as an inhibitor of saline. Neither exposure to sucrose-lemon before saline-lemon nor alternating exposure to sucrose and saline alone had the same effect. These results provide support for an associative theory of perceptual learning that suggests that exposure to complex stimuli aids later discrimination partially as a result of establishing inhibitory associations between their unique elements.
\end{abstract}

Exposure to two complex stimuli will increase the extent to which they can later be distinguished. Consider rats that are exposed to solutions of lemon-sucrose and lemon-saline and then receive $\mathrm{LiCl}$ after consuming one of them. The aversion will generalize less strongly to the other solution than if there had been no prior exposure (e.g., Mackintosh, Kaye, \& Bennett, 1991; Symonds \& Hall, 1995). The schedule of this exposure is also important. Symonds and Hall reported that alternating exposure to the two compound solutions reduced generalization between them more than did the same total exposure given in blocks in which all exposures to one solution preceded exposure to the other (see also Bennett \& Mackintosh, 1999).

In order to explain this result, Symonds and Hall (1995) invoked Gibson's (1969) suggestion that the opportunity to compare and contrast two stimuli would attract attention to their unique features at the expense of the common features that do not differentiate them. An alternative analysis is suggested by the associative theory of perceptual learning put forward by McLaren, Kaye,

\footnotetext{
The experimental work for this paper took place while the first author was at the University of Cambridge, and he thanks Churchill College, Cambridge, for its support of the author as a graduate student and as a research fellow. Correspondence concerning this article should be addressed to N. J. Mackintosh, Department of Experimental Psychology, University of Cambridge, Downing Site, Cambridge CB2 3EB, England (e-mail: n.mackintosh@ psychol.cam.ac.uk).
}

and Mackintosh (1989; see McLaren \& Mackintosh, 2000 , for a recent review). This account suggests that over the course of alternating exposure, the common element, lemon, will form excitatory associations with the unique elements, sucrose and saline. Presentation of lemon-sucrose will thus retrieve the representation of the absent saline, and presentation of lemon-saline will retrieve the representation of the absent sucrose. This is exactly the situation that would establish the presented cue as a conditioned inhibitor of the absent but retrieved one. One would therefore expect mutual inhibitory associations to be formed between sucrose and saline. During blocked exposure, one would also expect the common element, lemon, to form excitatory associations with the unique elements, sucrose and saline. But in the absence of alternation, the presence of one of the unique elements does not reliably predict the absence of the other, so no mutually inhibitory links should be formed between sucrose and saline.

Several studies have provided indirect evidence consistent with this inhibitory analysis. For example, Espinet, Iraola, Bennett, and Mackintosh (1995) showed that after alternating exposure to two compound stimuli (AX and BX), training A as a predictor of some unconditioned stimulus (US) will allow B to pass both summation and retardation tests as an inhibitor of that USthe Espinet effect. This result has been replicated both with rats (Artigas, Chamizo, \& Peris, 2001, Experiments 1A, 1B, and 1C) and with humans (Artigas et al. 2001, 
Experiments 2 and 3; Graham, 1999). Espinet et al.'s experiments were prompted by the suggestion from McLaren et al. (1989) that alternating exposure to AX and BX should establish inhibitory links between their unique elements. But Espinet et al. noted that it was not clear how that mutual inhibition between $A$ and $B$ would allow $B$ to act as a conditioned inhibitor of a US that was subsequently paired with A. Although some progress has been made on this issue (see Artigas et al., 2001; Bennett, Scahill, Griffiths, \& Mackintosh, 1999), it remains true that this approach can provide only indirect evidence of the supposed mutual inhibition between $A$ and B. However, the first direct evidence for the formation of inhibitory associations between the unique elements of two compound stimuli presented in alternation was provided by Dwyer, Bennett, and Mackintosh (2001).

If alternating exposure to sucrose-lemon and salinelemon does establish inhibitory associations between sucrose and saline, it should retard the formation of an excitatory, within-compound association when sucrose and saline are subsequently presented together. The strength of this association was assessed by inducing a sodium appetite, which causes an increase in the consumption both of saline and of flavors associated with saline. In two experiments, sodium depletion increased consumption of sucrose more in groups given blocked exposure than in those given alternating exposure. Although such retardation of excitatory conditioning is consistent with the existence of inhibitory associations, it has usually been thought that evidence from both retardation and summation tests would be needed to provide a conclusive demonstration of inhibitory conditioning. Dwyer et al. (2001) were unable to provide evidence from such a summation test. Thus, the first aim of the present study was to find evidence from a summation test to complement the previous finding that alternating exposure produces a retardation of excitatory conditioning.

\section{EXPERIMENT 1}

Animals were given either alternating (Group ALT) or blocked (Group BLK) exposure to sucrose-lemon and saline-lemon before all the animals received peppermint paired with saline. Finally, all the animals were tested for their consumption of a compound of peppermint and sucrose both under sodium depletion and under control conditions. Sodium depletion should act to increase the consumption of the peppermint previously associated with saline. If alternating exposure to sucrose-lemon and saline-lemon establishes sucrose as an inhibitor of saline, the effect of sodium depletion should be attenuated in Group ALT, but not in Group BLK. This test does differ from the usual summation test, in which reaction to the $\mathrm{CS}+$ is compared with the reaction to a compound of the $\mathrm{CS}+$ and the putative inhibitor. However, rodents show a high unconditioned preference for sucrose (Beauchamp \& Mason, 1991), so using peppermint-sucrose as the test solution for all groups ensured that no complications were introduced by having to compare the consumption of solutions of different palatability. This test also differed from our previous attempts at a summation test in that peppermint, rather than quinine, was used as the $\mathrm{CS}+$. Quinine is not readily consumed by rodents, even at low concentrations (Beauchamp \& Mason, 1991). It was hoped that the use of a more neutral cue solution would improve the sensitivity of the test.

\section{Method}

\section{Subjects and Apparatus}

Thirty-two naive male hooded Lister rats were used as the subjects of the first replication of Experiment 1. For reasons explained below, this experiment was replicated with a further 16 animals. All the animals were supplied by Charles River Limited, Kent, U.K. Those in the first replication weighed between 240 and $280 \mathrm{~g}$ at the start of the experiment, whereas those in the second weighed between 290 and $360 \mathrm{~g}$. All were housed in groups of 4 under a 12:12-h light:dark cycle. They were maintained on ad-lib food and a water deprivation schedule that allowed 1 - $\mathrm{h}$ access to water, $1 \mathrm{~h}$ after the last experimental session each day. The animals were divided into equal groups, so there were 16 animals per group in Replication 1 and 8 per group in Replication 2.

Training and testing took place in a separate experimental room that contained eight acrylic drinking boxes, $32 \times 15 \times 12 \mathrm{~cm}$, with smooth flat flooring of the same material as the walls and wiremesh lids. A 50-ml drinking bottle with a metal spout could be inserted at one end of each box. Consumption was assessed by weighing the bottles before and after each session. The cue solutions used in the experiment were $0.5 \%$ v/v peppermint (Supercook, Sherburnin-Elmet, Leeds, U.K.), $0.9 \% \mathrm{w} / \mathrm{v}$ saline, $2.0 \% \mathrm{w} / \mathrm{v}$ sucrose, and $2.0 \%$ v/v Sainsbury's pure lemon juice.

\section{Procedure}

Pretraining. Following initial water deprivation, all the animals received four sessions of preliminary training, two a day for 2 days, on each of which they were placed in the drinking cages for $10 \mathrm{~min}$ with unlimited access to water. Throughout the experiment, the morning session began at 10:00, and the afternoon session began at 15:00.

Exposure. All the rats then received 16 exposure sessions, 2 sessions per day for 8 days, on which they were given $8 \mathrm{ml}$ of either sucrose-lemon or saline-lemon to drink. Each session lasted for a minimum of $10 \mathrm{~min}$ and lasted longer if a rat did not consume all $8 \mathrm{ml}$ of the fluid. Half of the rats in Group ALT drank saline-lemon in the morning and sucrose-lemon in the afternoon on odd days and drank the solutions in the reverse order on even days; the other half received the solutions in the opposite order. Half of the rats in Group BLK received 8 sessions of sucrose-lemon, followed by 8 of saline-lemon; the other half received the solutions in the opposite order.

Treatment and test. Following exposure, all the animals received four pairings of saline and peppermint, two sessions per day for 2 days. As during pretraining, each session lasted for a minimum of $10 \mathrm{~min}$ and lasted longer if a rat did not consume all $8 \mathrm{ml}$ of the fluid.

After this training was completed, a 6-day testing procedure began. On the morning of Day 1, acute sodium depletion was induced in half of the animals in each group. They were injected (i.p.) with a total of $10 \mathrm{mg} / \mathrm{kg}$ of furosimide in two injections spaced $1 \mathrm{~h}$ apart; animals not depleted remained in their home cages. Before these injections, the animals' diet was switched from ordinary rat chow to a salt-free diet. Water was made available to all the animals for $6 \mathrm{~h}$ after the second injection. On Day 2, all the animals were given a 10 -min test session, with unlimited access to a compound 
of sucrose and peppermint. After the test session, all the animals were allowed ad-lib access to ordinary rat chow and water until the afternoon of Day 4, when the water was removed from all the animals. On the morning of Day 5, acute sodium depletion was induced in the animals that had not received this treatment on Day 1. As on Day 1, the animals' diet was switched from ordinary rat chow to a salt-free diet before the first injection, and water was made available to all animals for $6 \mathrm{~h}$ after the second injection. Finally, on Day 6 , all the animals were given a 10-min test session, with unlimited access to a compound of sucrose and peppermint. Thus, all the animals were tested for their consumption of the sucrose-peppermint compound, both while sodium depleted and while nondepleted.

For reasons that remain unclear, 5 animals in the first replication (4 from Group ALT and 1 from Group BLK) consumed less than $3 \mathrm{ml}$ when tested under sodium depletion. Such low consumption is normally an indication of a blockage in the drinking spouts, although it is most unusual for so many spouts to become blocked at once. To confirm that any difference between groups was not due to this problem, these animals were removed from the statistical analysis, and as was noted above, the experiment was replicated. There were no differences between the two replications, with the exception that the second replication used only half the number of animals per group.

\section{Results and Discussion}

There was some evidence of a neophobic reaction to the saline-lemon and sucrose-lemon compounds on the initial exposure trials, but after this, all of the solutions offered were consumed.

To obtain an indication of the effect of sodium depletion on the consumption of the peppermint-sucrose compound, difference scores were calculated, with the amount consumed when the animals were nondepleted subtracted from the amount consumed when they were depleted. Figure 1 shows the average difference in consumption for Groups ALT and BLK for both replications. As was expected, the animals in Group BLK consumed more of the sucrose-peppermint compound when they were sodium depleted. This was not true of the animals in Group ALT. These differences were not produced by variation in consumption when the animals were nondepleted, although there was a difference in absolute consumption between replications. When nondepleted, the animals in Group ALT consumed an average of $8.38 \mathrm{ml}(S E M=0.65)$ of the peppermint-sucrose compound in Replication 1, and $11.53 \mathrm{ml}(S E M=0.83)$ in Replication 2, whereas the animals in Group BLK consumed an average of $7.80 \mathrm{ml}(S E M=0.43)$ in Replication 1 and $10.93 \mathrm{ml}(S E M=0.78)$ in Replication 2 .

The difference in consumption between depleted and nondepleted tests was analyzed with a three-way analysis of variance (ANOVA) with factors of exposure, test order (depleted first, depleted second), and replication. This analysis excluded the low-drinking animals from the first replication. There was a significant effect of exposure $[F(1,35)=11.09]$. (A significance level of $p<$ .05 was adopted here and throughout this study.) There was no significant effect of either test order or replication $\left(F_{\mathrm{S}}<1\right)$, nor were there any significant interactions between factors $\left[F_{\mathrm{s}}(1,35)<1.68\right]$. A three-way ANOVA, using the same factors, of nondepleted consumption

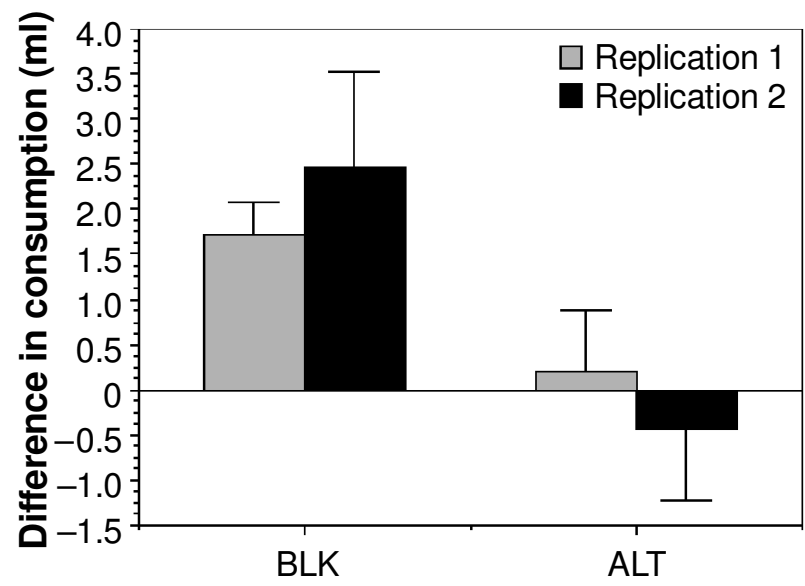

Figure 1. Mean difference in consumption (with $S E M$ ) of the peppermint-sucrose compound for both replications of Experiment 1 (consumption when the animals were depleted - consumption when they were nondepleted). The figure does not include data from animals with excessively low consumption on test in the first replication (see the text for details). Prior to test, the animals had been exposed to the compound flavors sucroselemon and saline-lemon and then had received four pairings of peppermint and saline. Group ALT received the compound solutions in alternation. Group BLK received the same amount of exposure in blocks, so that all presentations of one compound preceded presentations of the other.

confirmed that there was a difference between replications $[F(1,35)=22.45]$. Importantly, there were no significant effects of exposure or test order $(F \mathrm{~s}<1)$ and no significant interactions between factors $[F \mathrm{~s}(1,35)<$ $1.88]$.

The results of this analysis are not dependent on the omission of the low-drinking animals from the first replication, for if those animals are included, there is still a significant effect of exposure $[F(1,40)=13.82]$. If anything, the numerical difference between Group BLK $(2.20, S E M=0.46)$ and Group ALT $(-1.23$, SEM $=$ 0.70 ) is larger with these animals included. The inclusion of these animals does not result in there being a significant effect of either test order or replication $\left(F_{\mathrm{s}}<\right.$ $1)$, nor were there any significant interactions between factors $\left[F_{\mathrm{S}}(1,40)<2.6\right]$. The critical result is that in Group BLK, depletion significantly increased the consumption of the sucrose-peppermint compound, whereas in Group ALT it did not. This is exactly the result that one would expect if alternating exposure to sucrose-lemon and saline-lemon establishes inhibitory associations between sucrose and saline. Sodium depletion should increase consumption of the peppermint previously paired with saline, but this effect would be counteracted by the inhibitory association between sucrose and saline in Group ALT. In Group BLK, there should be no inhibitory association between sucrose and saline, and thus one would expect to find that sodium depletion would increase consumption of the sucrose-peppermint compound.

In short, Experiment 1 provides the first direct evidence from a summation test that alternating exposure 
to two compounds sharing a common element will create inhibitory associations between the unique elements of the two compounds.

\section{EXPERIMENT 2}

Since Experiment 1 provided evidence from a summation test to complement the retardation tests that have previously been reported (Dwyer et al., 2001), it seemed reasonable to conclude that alternating exposure to two compounds will create inhibitory associations between their unique elements. What had not been unambiguously demonstrated was that the presence of a common flavor element during compound exposure is needed to establish these inhibitory links. Several earlier studies had provided evidence of the importance of such common elements in generating perceptual learning (Mackintosh et al., 1991, Experiment 4) and indirect evidence that they are important for inhibitory effects (Espinet et al., 1995, Experiment 3). In Experiment 2, we sought more direct evidence. One group of animals was given alternating exposure to saline and sucrose to see whether this would act to retard the later formation of withincompound associations between sucrose and saline in the same way that alternating exposure to sucrose-lemon and saline-lemon does.

Alternating exposure to sucrose-lemon and salinelemon should, according to standard associative theory, establish mutually inhibitory associations between sucrose and saline. But the results of Experiment 1 and those previously reported by Dwyer et al. (2001) implied that it is the inhibitory association from sucrose to saline that is critical. In Experiment 1, the pairing of peppermint and saline would be expected to increase consumption of peppermint in animals under sodium depletion. Group BLK did indeed show such an increase in consumption of sucrose-peppermint when depleted. The absence of any such effect in Group ALT suggested that in their case, the presence of sucrose prevented the retrieval of any representation of saline. In the Dwyer et al. experiments, the pairing of sucrose and saline could be expected to increase consumption of sucrose after sodium depletion. The smaller effect seen in Group ALT than in Group BLK is most simply explained by supposing that alternating exposure retarded the formation of an excitatory association between sucrose and saline, because sucrose had been established as an inhibitor of saline.

The second purpose of Experiment 2 was to provide more direct evidence that it was, indeed, the inhibitory association from sucrose to saline (and not that from saline to sucrose) that was responsible for our results. The method employed was similar to that used by Bennett et al. (1999; see also Graham, 1999) in a study of perceptual learning. They exposed rats each day to the same two solutions presented in the same order: either AX immediately followed by BX or BX immediately followed by AX. On the basis of a variety of Pavlovian experiments (e.g., Ewing, Larew, \& Wagner, 1985; Maier, Rapaport, \& Wheatley, 1976; Wagner \& Larew, 1985), this procedure should establish an inhibitory association between the second flavor and the first. It is true that when AX is presented, B should be retrieved, owing to its association with $\mathrm{X}$, which is the mechanism for the formation of an inhibitory link between $\mathrm{A}$ and $\mathrm{B}$, as was noted earlier. However, this would be counteracted by the excitatory connection between $\mathrm{A}$ and $\mathrm{B}$ produced by the fact that AX was followed by BX on each trial. After training with $\mathrm{AX}$ followed by BX, A was paired with a US. This established B as an inhibitor of that US in both rats (Bennett et al., 1999) and humans (Graham, 1999). The same was not true when BX had been presented in advance of AX. We employed the same exposure procedure, combined with the test procedure used in Dwyer et al. (2001), to provide a direct test of whether it was the inhibitory association from sucrose to saline or that from saline to sucrose that was responsible for the results of our earlier experiments. Thus, Experiment 2 included a group of animals for which exposure to sucrose-lemon was preceded on each day by saline-lemon. This should establish sucrose as an inhibitor of saline and, thus, interfere with the ability of sucrose to retrieve a representation of saline after the animals had been exposed to a sucrose-saline compound. A further group received preexposure to saline-lemon only after receiving sucroselemon each day. This should establish saline as an inhibitor of sucrose but should not affect sucrose's ability to retrieve a representation of saline.

\section{Method}

\section{Subjects and Apparatus}

The subjects were 32 male hooded Lister rats, divided into four equal groups, obtained from the same source as those in Experiment 1 . They weighed between 230 and $285 \mathrm{~g}$ at the start of the experiment and were housed and maintained as in Experiment 1. The apparatus and solutions were also the same.

\section{Procedure}

Pretraining. Following initial water deprivation, all the animals received six sessions of preliminary training, two a day for 3 days. During Session 1, they were placed in the drinking cages and given access to $4 \mathrm{ml}$ of water for $2 \mathrm{~min}$, immediately followed by a further $4 \mathrm{ml}$ for $10 \mathrm{~min}$. When it became clear that many of the animals were not consuming the full amount offered, a further four preliminary sessions were given, but with the volume presented reduced to $3 \mathrm{ml}$ per exposure. The animals readily consumed this amount.

Exposure. Following pretraining, the animals were randomly assigned to four equal groups: $\mathrm{AX} / \mathrm{BX}, \mathrm{A} / \mathrm{B}, \mathrm{AX} \rightarrow \mathrm{BX}$, and $\mathrm{BX} \rightarrow \mathrm{AX}$. All the rats then received 16 exposure sessions, 2 sessions per day for 8 days. An exposure session consisted of 2-min access to $3 \mathrm{ml}$ of one solution, followed by 10-min access to a further $3 \mathrm{ml}$ of a second solution. The animals in Group AX/BX were given the same solution during each session; half of the 8 rats in this group drank saline-lemon in the morning and sucrose-lemon in the afternoon on odd days, and in the reverse order on even days; the other half received the solutions in the opposite order. Group A/B was treated in exactly the same manner, except that the solutions presented to them were saline and sucrose alone. The animals in Group $\mathrm{AX} \rightarrow \mathrm{BX}$ always received $3 \mathrm{ml}$ of lemon-saline, followed by $3 \mathrm{ml}$ of lemonsucrose, whereas those in Group $\mathrm{BX} \rightarrow \mathrm{AX}$ received $3 \mathrm{ml}$ of lemonsucrose, followed by $3 \mathrm{ml}$ of lemon-saline.

Treatm ent and test. Following exposure, all the animals received a single pairing of sucrose and saline. The session lasted for 
a minimum of $10 \mathrm{~min}$ and lasted longer if a rat did not consume all $6 \mathrm{ml}$ of the fluid offered.

The day after this training session was completed, a 6-day testing procedure began. This was the same as that described in Experiment 1 , save that the test solution was sucrose alone. Thus all the animals were tested for their consumption of sucrose while sodium depleted and nondepleted.

\section{Results and Discussion}

With the exception of the first exposure trial, the rats consumed all of the fluid provided throughout exposure and during the sucrose-saline pairing. As in Experiment 1, difference scores were calculated, with the amount consumed when they were nondepleted subtracted from the amount consumed when they were depleted, in order to obtain an indication of the effect of sodium depletion on the consumption of sucrose. Figure 2 shows the average difference in consumption for all groups. Unlike in Experiment 1 , test order did have an effect on this score, so the figure shows differential consumption for each test order separately. The large positive difference scores for Groups $\mathrm{A} / \mathrm{B}$ and $\mathrm{BX} \rightarrow \mathrm{AX}$ indicate that sodium depletion markedly increased consumption of sucrose in those groups but had a smaller effect on Groups AX/BX and $\mathrm{AX} \rightarrow \mathrm{BX}$, which did not show such large difference scores. The difference scores were greater in the animals tested under sodium depletion first. These differences were not produced by variation in consumption when the animals were nondepleted. While nondepleted animals in Group AX/BX consumed an average of $10.56 \mathrm{ml}$

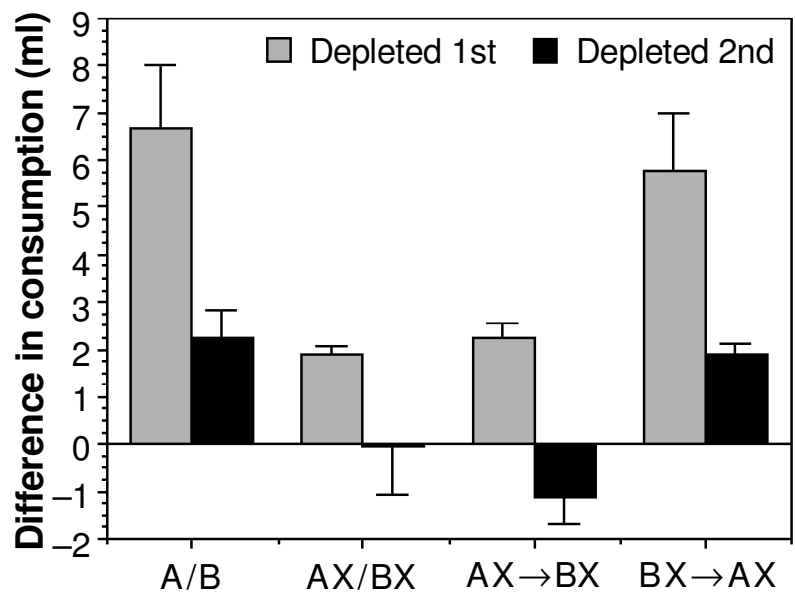

Figure 2. Mean difference in consumption (with $S E M$ ) of sucrose in Experiment 2 (consumption when the animals were depleted - consumption when they were nondepleted). Since there was an overall difference between test order, but no interaction with any other factor, group means are shown for both test orders separately: depleted on the first test, but nondepleted on the second test, and nondepleted on the first test, but depleted on the second test. Prior to test, Group A/B had received alternating exposure to sucrose and saline, Group $\mathrm{AX} / \mathrm{BX}$ had received alternating exposure to sucrose-lemon and saline-lemon, Group $\mathrm{AX} \rightarrow \mathrm{BX}$ had received saline-lemon immediately before sucroselemon, and Group $\mathrm{BX} \rightarrow \mathrm{AX}$ had received sucrose-lemon immediately before saline-lemon. All the groups received a single pairing of sucrose and saline.
$(S E M=0.85)$ of sucrose, Group $\mathrm{AX} \rightarrow \mathrm{BX}$ consumed $11.62 \mathrm{ml}(S E M=0.69)$, Group $\mathrm{BX} \rightarrow \mathrm{AX}$ consumed $10.63 \mathrm{ml}(S E M=0.49)$, and Group A/B consumed $10.22 \mathrm{ml}(S E M=0.48)$.

A two-way ANOVA, with factors of exposure type and test order, of nondepleted consumption confirmed that there were no significant effects of exposure type or test order or any interaction between the two $\left(F_{\mathrm{S}}<1\right)$. The difference in consumption between depleted and nondepleted tests was analyzed with a two-way ANOVA with the same factors as above. There were significant effects of exposure type $[F(3,24)=11.90]$ and test order $[F(1,24)=$ 35.03], but no significant interaction between them $(F<$ 1). Pairwise comparisons were made between groups, collapsed over test order, with the Type 1 error rate controlled by the Bonferroni method. These comparisons confirmed that Group A/B differed from Group AX/BX. This accords with the prediction from McLaren et al. (1989) that the formation of inhibitory associations by alternating exposure to two solutions depends on their sharing common elements. In addition, Group $\mathrm{BX} \rightarrow \mathrm{AX}$ was different from both Group AX/BX and Group $\mathrm{AX} \rightarrow \mathrm{BX}$, whereas the last two groups did not differ. This is consistent with the expectation that, of the mutually inhibitory associations between sucrose and saline created by intermixed exposure to sucrose-lemon and saline-lemon, only the inhibitory association from sucrose to saline would prevent sucrose from retrieving a representation of saline, whereas an inhibitory association from saline to sucrose would have no such effect.

\section{GENERAL DISCUSSION}

Experiment 1 provided evidence from a summation test that alternating exposure to sucrose-lemon and salinelemon will establish sucrose as an inhibitor of saline. This complements previously reported retardation tests (Dwyer et al., 2001). Taken together, they provide unambiguous proof that alternating exposure to two compound stimuli that have some common features will establish inhibitory links between the unique elements of those stimuli. The results of Experiment 2 demonstrated that alternating exposure to two stimuli will be less likely to establish inhibitory links between them if they share fewer common features. They also implied that while an inhibitory association from sucrose to saline will prevent sucrose from retrieving a representation of saline, an inhibitory association from saline to sucrose will not.

We must acknowledge that the retardation results from Experiment 2 are not supported by corresponding summation tests. However, the lack of a summation test does not seem seriously to question the conclusions stated above. First, the key results of Experiment 2 are those from treatments that did not produce a retardation effect. That is, alternating exposure to sucrose and saline alone did not result in a retardation of learning a sucrose-saline association, nor did consistently presenting sucrose-lemon before saline-lemon have an effect on sucrose consumption. Second, summation tests are normally thought to 
rule out the possibility that any retardation effect is being produced by latent inhibition. In the present case, only Group $\mathrm{AX} \rightarrow \mathrm{BX}$ showed a retardation effect for which there is not already a corresponding summation test. But Group $\mathrm{AX} \rightarrow \mathrm{BX}$ received the same amount of exposure to saline and sucrose as did Group $\mathrm{BX} \rightarrow \mathrm{AX}$, which did not show a retardation effect. Both groups received this exposure in compound with lemon, so that both groups experienced some sort of context change between preexposure and conditioning. In fact, the only difference between the two groups was the order in which sucroselemon and saline-lemon were presented during preexposure. There is no obvious reason why this should affect latent inhibition, but for the reasons elaborated in the introduction to Experiment 2, it should affect the direction of any inhibitory link formed between sucrose and saline.

The results of the present experiments and those of Dwyer et al. (2001) are not easily explained by the hypothesis that alternating preexposure allowed animals to compare the two compound solutions and that this enhanced the contrast between them (Gibson, 1969; Symonds \& Hall, 1995). Rescorla and Furrow (1977) have, indeed, demonstrated that the similarity of two stimuli will affect the ease with which they are associated when paired, so there is some reason to expect that making two stimuli more distinct could retard the formation of an association between them. However, there is no reason to suppose that sucrose and saline themselves become more discriminable after preexposure to sucrose-lemon and saline-lemon, since Mackintosh et al. (1991) have shown that it is the discrimination between the compounds that is facilitated by preexposure, not the discrimination between sucrose and saline alone. Moreover, the results of Experiment 2 provide no support for the Gibsonian analysis. Alternating exposure to sucrose and saline alone might have been expected to make them more distinct, and thus, to have the same effect as alternating exposure to the compounds. It did not. Nor is there any reason to expect that the order in which the animals were exposed to the two compound solutions should have affected their discriminability. But it did affect the results of the retardation test.

Prior to Dwyer et al. (2001) and the present study, the bulk of the research aimed at assessing the application of McLaren et al.'s (1989) model to perceptual learning has been conducted with designs that were only indirectly applicable to the substance of that theory (Artigas et al., 2001; Bennett et al., 1999; Espinet et al., 1995; Graham, 1999; Mackintosh et al., 1991). Direct confirmation that intermixed exposure to two complex stimuli will create inhibitory links between their unique elements is of importance not only in itself, but also because the existence of these inhibitory links is assumed by McLaren et al.'s (1989) model in its explanation of perceptual learning.

The finding that exposure to saline followed by sucrose (in Group $\mathrm{AX} \rightarrow \mathrm{BX}$ ) was more effective than exposure to sucrose followed by saline (in Group $\mathrm{BX} \rightarrow \mathrm{AX}$ ) in retarding the formation of an excitatory association be- tween sucrose and saline is consistent with earlier studies of inhibition in Pavlovian conditioning (e.g., Ewing et al., 1985; Maier et al., 1976; Wagner \& Larew, 1985). But it is also consistent with earlier experiments on perceptual learning in flavor aversion conditioning. The Espinet effect, which provides indirect evidence of inhibitory associations between the unique elements of compound flavors, also appears only in subjects exposed to $\mathrm{AX}$ followed by $\mathrm{BX}$, rather than vice versa (Artigas et al., 2001; Bennett et al., 1999; Graham, 1999). Moreover, Bennett et al. found that generalization to BX of an aversion conditioned to AX (a direct measure of perceptual learning) was more profoundly affected by exposure to $\mathrm{AX}$ followed by BX than vice versa. They presented evidence to show that the main reason for such generalization in animals given no prior exposure to both $\mathrm{AX}$ and $\mathrm{BX}$ compounds was because, on test trials with BX, $X$ retrieved a representation of the previously conditioned A, from which it would follow that the most effective way of reducing such generalization would be by establishing B as an inhibitor of A.

In summary, when considered along with the results of Dwyer et al. (2001), the present results provide direct and compelling evidence that alternating exposure to two compound flavors will produce inhibitory associations between their unique elements. In addition, they confirm that for such inhibition to arise, the exposed flavors must share some common elements. As such, they strongly support suggestions drawn from McLaren et al. (1989) and McLaren and Mackintosh (2000)—namely, that the formation of inhibitory associations between unique elements is one of the mechanisms underlying perceptual learning effects and that the same inhibitory associations also underpin the Espinet effect.

\section{REFERENCES}

Artigas, A. A., Chamizo, V. D., \& Peris, J. M. (2001). Inhibitory associations between neutral stimuli: A comparative approach. Animal Learning \& Behavior, 29, 46-65.

Beauchamp, G. K., \& Mason, J. R. (1991). Comparative hedonics of taste. In R. C. Bolles (Ed.), The hedonics of taste (pp. 159-184). Hillsdale, NJ: Erlbaum.

Bennett, C. H., \& Mackintosh, N. J. (1999). Comparison and contrast as a mechanism of perceptual learning? Quarterly Journal of Experimental Psychology, 52B, 253-272.

Bennett, C. H., Scahill, V. L., Griffiths, D. P., \& Mackintosh, N. J. (1999). The role of inhibitory associations in perceptual learning. Animal Learning \& Behavior, 27, 333-345.

Dwyer, D. M., Bennett, C. H., \& Mackintosh, N. J. (2001). Evidence for inhibitory associations between the unique elements of two compound flavors. Quarterly Journal of Experimental Psychology, 54B, 97-109.

Espinet, A., Iraola, J. A., Bennett, C. H., \& Mackintosh, N. J. (1995). Inhibitory associations between neutral stimuli in flavoraversion conditioning. Animal Learning \& Behavior, 23, 361-368.

Ewing, M. F., Larew, M. B., \& Wagner, A. R. (1985). Distributionof-trials effects in Pavlovian conditioning: An apparent involvement of inhibitory backward conditioning with short intertrial intervals. Journal of Experimental Psychology: Animal Behavior Processes, 11, 537-547.

Gibson, E. J. (1969). Principles of perceptual learning and development. New York: Appleton-Century-Crofts. 
Graham, S. (1999). Retrospective revaluation and inhibitory associations: Does perceptual learning modulate our perception of the contingencies between events? Quarterly Journal of Experimental Psychology, 52B, 159-185.

Mackintosh, N. J., Kaye, H., \& Bennett, C. H. (1991). Perceptual learning in flavor aversion conditioning. Quarterly Journal of Experimental Psychology, 43B, 297-322.

Maier, S. F., Rapaport, P., \& Wheatley, K. L. (1976). Conditioned inhibition and the UCS-CS interval. Animal Learning \& Behavior, 4, 217-220.

McLaren, I. P. L., Kaye, H., \& Mackintosh, N. J. (1989). An associative theory of the representation of stimuli: Applications to perceptual learning and latent inhibition. In R. G. M. Morris (Ed.), Parallel distributed processing: Implications for psychology and neurobiology (pp. 102-130). Oxford: Oxford University Press, Clarendon Press.

McLaren, I. P. L., \& Mackintosh, N. J. (2000). An elemental model of associative learning: I. Latent inhibition and perceptual learning. Animal Learning \& Behavior, 28, 211-246.

Rescorla, R. A., \& Furrow, D. R. (1977). Stimulus similarity as a determinant of Pavlovian conditioning. Journal of Experimental Psychology: Animal Behavior Processes, 3, 203-215.

Symonds, M., \& Hall, G. (1995). Perceptual learning in flavor aversion conditioning: Roles of stimulus comparison and latent inhibition of common elements. Learning \& Motivation, 26, 203-219.

WAGNer, A. R., \& LAREW. M. B. (1985) Opponent processes and Pavlovian inhibition. In R. R. Miller \& N. E. Spear (Eds.), Information processing in animals: Conditioned inhibition (pp. 223-267). Hillsdale, NJ: Erlbaum.

(Manuscript received February 20, 2002; accepted for publication May 6, 2002.) 